\title{
Analysis on parameter switching methods for hydropower dominant system
}

\author{
Shengjing She ${ }^{1, *}$, Chongtao $\mathrm{Li}^{1}$, Yiping Chen ${ }^{2}$, Rongzhao $\mathrm{Yang}^{3}$, Liang $\mathrm{Xiao}^{2}$, Kehan Zeng ${ }^{1}$ and Qin $\mathrm{Gao}^{2}$ \\ ${ }^{1}$ Shaanxi Key Laboratory of Smart Grid, Xi' an Jiaotong University, 710049, Xi'an, Shaanxi, China \\ ${ }^{2}$ Power Dispatching and Control Center, China Southern Power Grid, 510663, Guangzhou, Guangdong, China \\ ${ }^{3}$ Electric Power Research Institute, China Southern Power Grid, 510663, Guangzhou, Guangdong, China
}

\begin{abstract}
With the rapid development of DC asynchronous interconnection, in order to ensure the frequency quality, higher requirements are put forward for the primary frequency control of hydropower units. In the high proportion hydropower system, it is difficult for the governor parameters to meet the requirements of primary frequency control and dynamic stability simultaneously. In order to solve the problem, this paper proposes a recommended parameter switching method by referring to the Nordic system. The parameter optimization methods before and after switching are proposed, and the effectiveness of the method is verified by time domain simulation.
\end{abstract}

\section{Introduction}

Frequency reflects the balance between power generation and electricity consumption and is an important index to be controlled in the operation of power system. In order to ensure the frequency characteristic of the system, the governor of hydropower unit, as the control mechanism of primary frequency modulation, is usually required to ensure that the unit has sufficient primary frequency control performance in parameters setting. Therefore, the governor parameters of the unit are set in some power plants to be extremely sensitive to the grid frequency deviation, thus the unit can quickly and fully make the frequency back when the frequency deviation occurs.

However, with the development of DC asynchronous interconnection, in the high-proportion hydropower system, the governor parameters are too sensitive and the water hammer effect of the hydropower unit is easy to provide negative damping, which causes the ultra-low frequency oscillation and seriously affects the safe and stable operation. Therefore, how to adjust the parameters of the hydropower unit governor to make it take into account the speed and dynamic stability of the primary frequency control at the same time has become an urgent key technical problem to be solved.

In reference [1], the parameters of the main unit are set to $1 / 4$ of the original value in view of the frequency oscillation in Yunnan asynchronous interconnection test. In references [2-3], governor parameters are optimized to suppress the ultra-low frequency oscillation. Nevertheless, in each of the above references, the regulating performance is sacrificed to ensure the stability. There are also some references trying to coordinate and optimize the parameters. In the [4-5], some parameter optimization methods have been proposed, considering both performance and stability.

However, the performance of primary frequency control is contradictory to the stability. At present, the parameters of the governor may not meet the requirements. In order to solve the problem, this paper refers to the two kinds of parameter switching methods of the governor of hydropower units in the Nordic system from [6], carries out comparative analysis and puts forward the recommended parameter switching method, so as to suppress ultra-low frequency oscillation from the side of the power plant. The parameter optimization methods before and after switching are proposed, and the effectiveness of the methods are verified by time domain simulation.

\section{The status of primary frequency control in Nordic power system}

The energy structure of Nordic power system is similar to that of Yunnan system in China. Both of them are high-proportion hydropower systems with frequency oscillation phenomenon. Therefore, it is of great significance to study the primary frequency control of hydropower units in Nordic power system. An introduction to Nordic system will be provided in this section.

\subsection{FCR in Nordic power system}

The frequency containment reserve(FCR) includes FCR for normal operation(FCR-N) and for disturbances(FCRD). FCR- $\mathrm{N}$ and $-\mathrm{D}$ are respectively activated within the range of $49.9 \sim 50.1 \mathrm{~Hz}$ and $49.5 \sim 49.9 / 50.1 \sim 50.5 \mathrm{~Hz}$. FCR-

Corresponding author: sheshengjing9511@126.com 
Table 1. FCR Requirements in the Nordic System

\begin{tabular}{|c|c|c|}
\hline Type & Range & Performance \\
\hline FCR-N & $49.9 \sim 50.1 \mathrm{~Hz}$ & within $2 \sim 3 \mathrm{~min}$ \\
\hline \multirow{2}{*}{ FCR-D } & $49.5 \sim 49.9 \mathrm{~Hz} /$ & $50 \%$ within $5 \mathrm{~s}$, and \\
& $50.1 \sim 50.5 \mathrm{~Hz}$ & $100 \%$ within $30 \mathrm{~s}$ \\
\hline
\end{tabular}

$\mathrm{N}$ is used to deal with random power unbalance during normal operation, while FCR-D is used to balance the power unbalance under large disturbance.

According to the performance index in [7], the output of FCR-N is required to be $63 \%$ within 60 s and $100 \%$ within $2 \sim 3$ minutes. FCR-D is required to produce $50 \%$ of its output within $5 \mathrm{~s}$ and $100 \%$ within $30 \mathrm{~s}$ in [8], as shown in Table 1.

\subsection{Switching methods of FCR-N/D}

In order to ensure primary frequency control reserve capacity under large disturbances, most hydropower units in Nordic power system need to provide both FCR$\mathrm{N}$ and -D services (denoted as FCR-N/D) from [9], while a hydropower unit has only one servo system and one turbine. To this end, since the end of 2014, two FCRN/D implementation methods have been proposed:

1. Conventional method: With a regulator structure, the parameters of FCR-N and FCR-D switch back and forth as the frequency deviation changes. This is the conventional mode still in use today, as shown in Fig. 1.

The PID parameters and droop coefficient $b_{p}$ of FCR$\mathrm{N}$ and $-\mathrm{D}$ are switched at the threshold $\Delta f= \pm 0.1 \mathrm{~Hz}$. While $|\Delta f| \geq 0.1 \mathrm{~Hz}$, the parameters of FCR-D are adopted; while $|\Delta f|<0.1 \mathrm{~Hz}$, the parameters of FCR-N are adopted.

2. Parallel method: There are two parallel governor structures, one for FCR-N and another for FCR-D, the frequency deviation input of two PID links is respectively added with saturation and dead zone, there is no parameter change in each link, as shown in Fig. 2.

In this paper, the conventional and parallel methods are analyzed. FCR-N and -D represent the units with stable parameters and sensitive parameters respectively, and FCR-N/D represents the units with switching parameters.

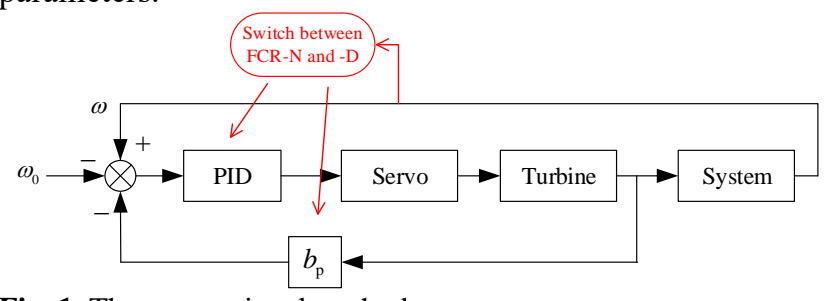

Fig. 1. The conventional method

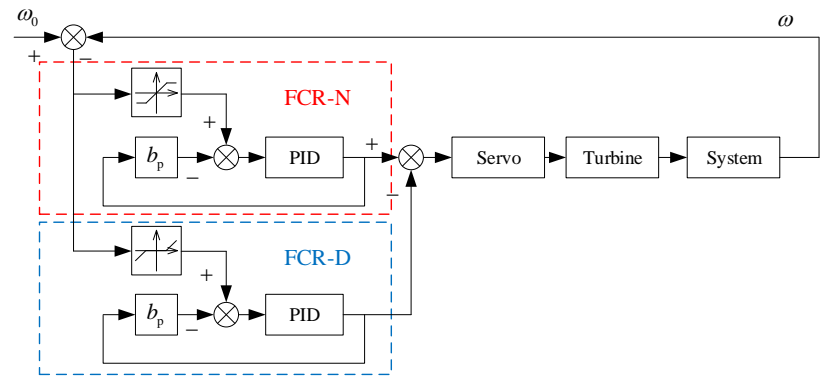

Fig. 2. The parallel method

\section{Analysis on the parameter switching methods}

The frequency characteristic of the power system depends largely on the performance of the primary frequency control. This section will make a detailed comparative analysis of these two methods, so as to get the recommended parameter switching method for hydropower unit in high-proportion hydropower system, ignoring the effect of dead zones.

\subsection{Analysis model}

\subsubsection{Speed regulation model}

The turbine governing system adopted is composed of three parts: the speed regulating control link, the servo system and the turbine. The nonlinearity of the turbine is ignored. The transfer function block diagram is shown in Fig. 3.

In Fig. 3, $\Delta \omega$ is the frequency deviation; $K_{\mathrm{P}}, K_{\mathrm{D}}$ and $K_{\mathrm{I}}$ are respectively proportional, differential and integral coefficient. $T_{\mathrm{D}}$ is the differential time constant; $b_{\mathrm{p}}$ is the droop coefficient; $T_{\mathrm{y}}$ is the time constant of the servo; $T_{\mathrm{w}}$ is the turbine time constant.

\subsubsection{The SFR model}

The frequency characteristics with different parameter switching methods are analyzed through the system frequency response(SFR) model, as shown in Fig. 4.

In Fig. $4, H$ is the equivalent inertia, $D$ is the equivalent damping, $\Delta P_{\mathrm{d}}$ is the power disturbance, $\Delta f$ is system frequency deviation, $k_{\mathrm{FCR}-\mathrm{N}}, k_{\mathrm{FCR}-\mathrm{D}}$ and $k_{\mathrm{FCR}-\mathrm{N} / \mathrm{D}}$ respectively represent the capacity proportion of units with stable, sensitive and switching parameters, $G_{\mathrm{FCR}}$ $\mathrm{N}(s), G_{\mathrm{FCR}-\mathrm{D}}(s)$ and $G_{\mathrm{FCR}-\mathrm{N} / \mathrm{D}}(s)$ represent the speed control transfer function of these three units.

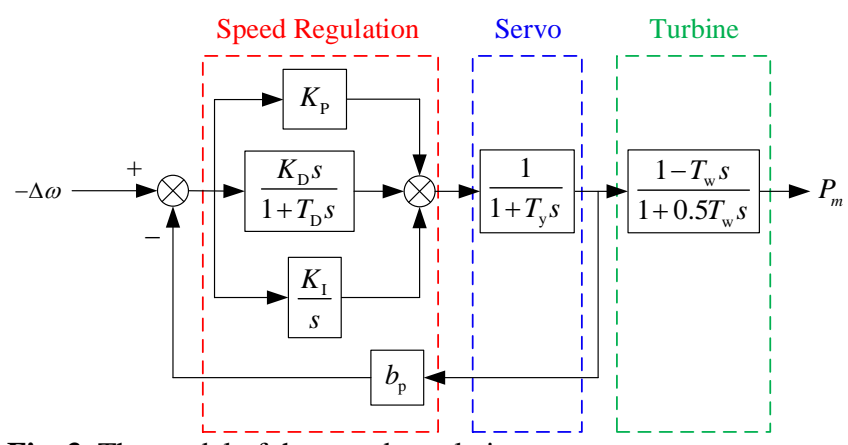

Fig. 3. The model of the speed regulating system

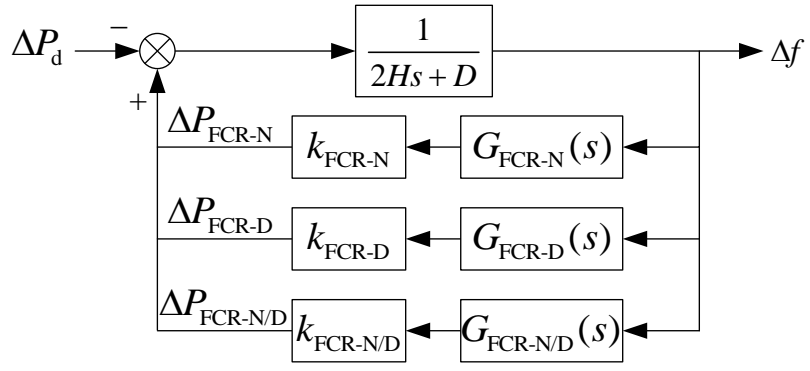

Fig. 4. The SFR model for analysis 
When the system frequency deviation is within the dead zone, FCR-D has no effect. When the system frequency deviation exceeds the dead zone, the input of FCR-N is constant, and in the conventional method, FCR-N/D is switched to the parameters of FCR-D. The system under small perturbation can be described by piecewise linear system model as:

$$
\Delta \dot{\boldsymbol{x}}(\boldsymbol{t})=\left\{\begin{array}{lc}
\boldsymbol{A}_{0} \Delta \boldsymbol{x}(\boldsymbol{t})+\boldsymbol{B}_{0} \Delta \boldsymbol{P}_{\mathrm{d}}(\boldsymbol{t}), & \Delta f<-0.1 \mathrm{~Hz} \\
\boldsymbol{A}_{1} \Delta \boldsymbol{x}(\boldsymbol{t})+\boldsymbol{B}_{1} \Delta \boldsymbol{P}_{\mathrm{d}}(\boldsymbol{t}), & |\Delta f| \leq 0.1 \mathrm{~Hz} \\
\boldsymbol{A}_{2} \Delta \boldsymbol{x}(\boldsymbol{t})+\boldsymbol{B}_{2} \Delta \boldsymbol{P}_{\mathrm{d}}(\boldsymbol{t}), & \Delta f>0.1 \mathrm{~Hz}
\end{array}\right.
$$

where the $\Delta \boldsymbol{x}(\boldsymbol{t})$ is the system state variables, $\Delta \boldsymbol{P}_{\mathrm{d}}$ is the input variables, $\boldsymbol{A}_{0}, \boldsymbol{A}_{1}, \boldsymbol{A}_{2}$ are the state matrices, $\boldsymbol{B}_{0}, \boldsymbol{B}_{1}$ and $\boldsymbol{B}_{2}$ are the input matrices.

\subsection{Problems with switching parameters}

First, the default parameters from [6] are used for analysis on the conventional method and parallel method.

In the conventional method, FCR-N and -D are realized by parameter switching in the same speed regulating system, without saturation and dead zone. When the frequency deviation exceeds the threshold, the governor is switched from FCR-N parameters to FCR-D parameters, and the frequency deviation input of FCR-D is equivalent to the enhanced dead zone [10]. When $K_{\mathrm{I}}$ is not zero, the expression of the power frequency characteristic in the conventional method can be written as:

$$
\Delta P_{\mathrm{G}}=\left\{\begin{array}{cc}
-\frac{\Delta f / f_{0}}{b_{\mathrm{p}, \mathrm{FCR}-\mathrm{N}}} & |\Delta f|<0.1 \mathrm{~Hz} \\
-\frac{\Delta f / f_{0}}{b_{\mathrm{p}, \mathrm{FCR}-\mathrm{D}}} & |\Delta f| \geq 0.1 \mathrm{~Hz}
\end{array}\right.
$$

where $f_{0}$ is the nominal frequency, $b_{\mathrm{p}, \text { FCR-N }}$ and $b_{\mathrm{p}, \text { FCR-D }}$ are the droop coefficients of FCR-N and $-\mathrm{D}, \Delta P_{\mathrm{G}}$ is active power increment of the generator.

The power frequency characteristic is continuous, providing a frequency equilibrium point for any disturbance when $b_{\mathrm{p}, \mathrm{FCR}-\mathrm{N}}=b_{\mathrm{p} \text {,FCR-D. Otherwise, the power }}$ frequency characteristic is discontinuous.

When the parallel method is adopted, due to the effect of saturation and dead zone, the power frequency characteristic is expressed as follows:

$$
\Delta P_{\mathrm{G}}=\left\{\begin{array}{cl}
-\frac{\Delta f / f_{0}}{b_{\mathrm{p}, \mathrm{FCR}-\mathrm{D}}}+\frac{0.1 / f_{0}}{b_{\mathrm{p}, \mathrm{FCR}-\mathrm{N}}}-\frac{0.1 / f_{0}}{b_{\mathrm{p}, \mathrm{FCR}-\mathrm{D}}} & \Delta f<-0.1 \mathrm{~Hz} \\
-\frac{\Delta f / f_{0}}{b_{\mathrm{p}, \mathrm{FCR}-\mathrm{N}}} & |\Delta f| \leq 0.1 \mathrm{~Hz}(3) \\
-\frac{\Delta f / f_{0}}{b_{\mathrm{p}, \mathrm{FCR}-\mathrm{D}}}-\frac{0.1 / f_{0}}{b_{\mathrm{p}, \mathrm{FCR}-\mathrm{N}}}+\frac{0.1 / f_{0}}{b_{\mathrm{p}, \mathrm{FCR}-\mathrm{D}}} & \Delta f>0.1 \mathrm{~Hz}
\end{array}\right.
$$

In parallel method, the power frequency characteristic is always continuous, providing an equilibrium point for any disturbance.

Next, the frequency characteristics of conventional

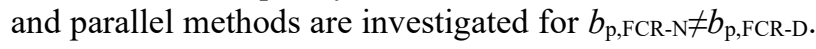

1) $b_{\mathrm{p}, \mathrm{FCR}-\mathrm{N}}<b_{\mathrm{p}, \mathrm{FCR}-\mathrm{D}}$

According to [6], suppose that $b_{\mathrm{p}, \mathrm{FCR}-\mathrm{N}}=0.04$ and $b_{\text {p,FCR-D }}=0.08$. The power frequency characteristic is discontinuous at the threshold $\Delta f= \pm 0.1 \mathrm{~Hz}$, as shown in Fig. 5(a).
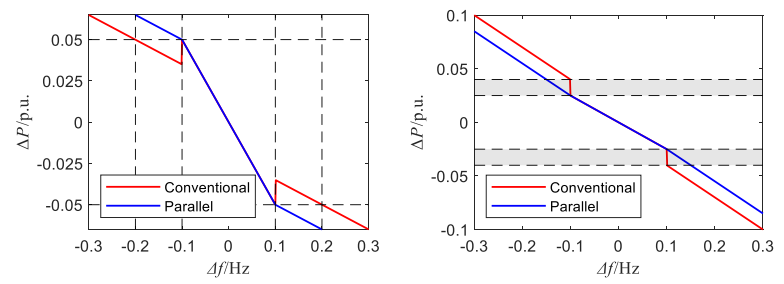

(a) $b_{\mathrm{p}, \mathrm{FCR}-\mathrm{N}}<b_{\mathrm{p}, \mathrm{FCR}-\mathrm{D}}$

(b) $b_{\mathrm{p}, \mathrm{FCR}-\mathrm{N}}>b_{\mathrm{p}, \mathrm{FCR}-\mathrm{D}}$

Fig. 5. The power frequency characteristics of the two methods

The frequency equilibrium point exceeds the dead zone will result in a sudden jump in the steady-state power value. Compared with parallel method, the steady-state frequency will have a significant drop or increase, as shown in Fig. 6.

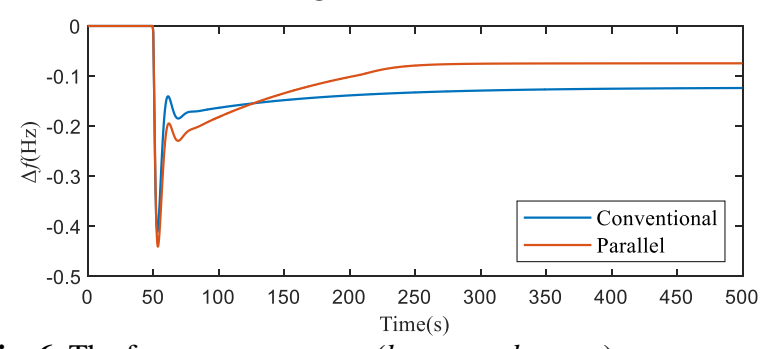

Fig. 6. The frequency response $\left(b_{\mathrm{p}, \mathrm{FCR}-\mathrm{N}}<b_{\mathrm{p}, \mathrm{FCR}-\mathrm{D})}\right.$

2) $b_{\mathrm{p}, \mathrm{FCR}-\mathrm{N}}>b_{\mathrm{p}, \mathrm{FCR}-\mathrm{D}}$

Suppose that $b_{\mathrm{p}, \mathrm{FCR}-\mathrm{N}}=0.08, b_{\mathrm{p}, \mathrm{FCR}-\mathrm{D}}=0.04$, the power frequency characteristic is discontinuous at the threshold $\Delta f= \pm 0.1 \mathrm{~Hz}$ in the conventional method, as shown in Fig. 5(b).

For some particular disturbances, the intersection of the load curve $\Delta P_{\mathrm{L}}$ and the generation curve $\Delta P_{\mathrm{G}}$ does not exist, as shown in Fig. 7.

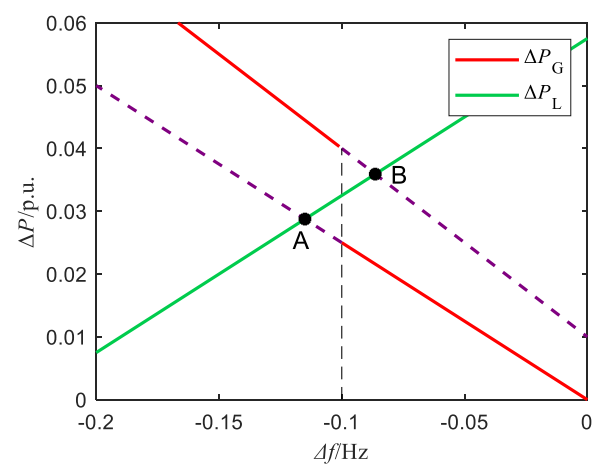

Fig. 7. The power frequency characteristic in the case of oscillation $\left(b_{\mathrm{p}, \mathrm{FCR}-\mathrm{N}}>b_{\mathrm{p}, \mathrm{FCR}-\mathrm{D})}\right.$

Assuming that the system can operate stably at point A, the piecewise frequency model according to equation (1) can be described as follows:

$$
0=\boldsymbol{A}_{1} \Delta \boldsymbol{x}(\boldsymbol{t})+\boldsymbol{B}_{1} \Delta \boldsymbol{P}_{\mathrm{d}}(\boldsymbol{t}), \quad|\Delta f| \leq 0.1 \mathrm{~Hz}
$$

However, the corresponding frequency $|\Delta f|>0.1 \mathrm{~Hz}$, which is not within the frequency range in formula (4), so the system cannot keep stably at point $\mathrm{A}$. In this case, $\Delta P_{\mathrm{G}}$ is greater than $\Delta P_{\mathrm{L}}$ and the frequency decreases, so the operating point will move to point $B$.

Similarly, the piecewise frequency model can be described as follows at point $\mathrm{B}$ :

$$
0=\boldsymbol{A}_{0} \Delta \boldsymbol{x}(\boldsymbol{t})+\boldsymbol{B}_{0} \Delta \boldsymbol{P}_{\mathrm{d}}(\boldsymbol{t}), \quad \Delta f<-0.1 \mathrm{~Hz}
$$

The corresponding frequency $\Delta f>-0.1 \mathrm{~Hz}$, which is not within the frequency range in formula (5), the system 
cannot keep stably at point B. $\Delta P_{\mathrm{L}}$ is greater than $\Delta P_{\mathrm{G}}$, the frequency rises, and the operating point will move to Point A, triggering the oscillation phenomenon near the dead zone, as shown in Fig. 8. The situation is similar to the frequency oscillation caused by the use of enhanced dead zone in the early stage of Yunnan asynchronous operation [10].

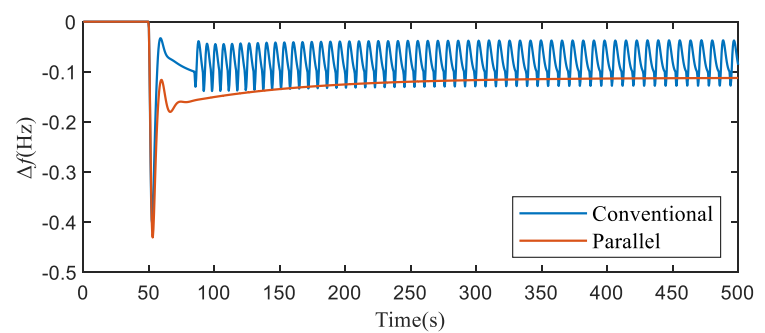

Fig. 8. The frequency response $\left(b_{\mathrm{p}, \mathrm{FCR}-\mathrm{N}}>b_{\mathrm{p}, \mathrm{FCR}-\mathrm{D})}\right)$

The power frequency characteristic of the parallel method is always continuous to provide an equilibrium point for any disturbance, which is different from the conventional method. When $b_{\mathrm{p}, \mathrm{FCR}-\mathrm{N}}<b_{\mathrm{p}, \mathrm{FCR} \text {-D }}$, the steadystate frequency of parallel method is higher than that of conventional method.

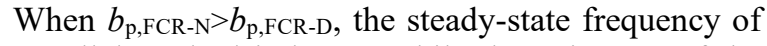
the parallel method is lower, while the existence of the frequency equilibrium is guaranteed and the frequency oscillation near the dead zone is avoided.

\subsection{Analysis on regulation performance}

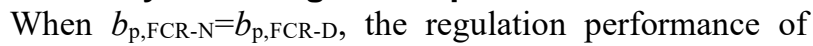
primary frequency control was simulated and analyzed in two different parameters switching methods.

In the case that the power disturbance is small and the frequency deviation does not exceed the dead zone, the power supports are provided by FCR-N in both methods, and the frequency responses are actually the same.

In the case of a large power disturbance, it is found by comparison that in the parallel method, the frequency deviation peak is larger and the frequency reaches the steady state more slowly, which means the regulation performance is worse, as shown in Fig. 9.

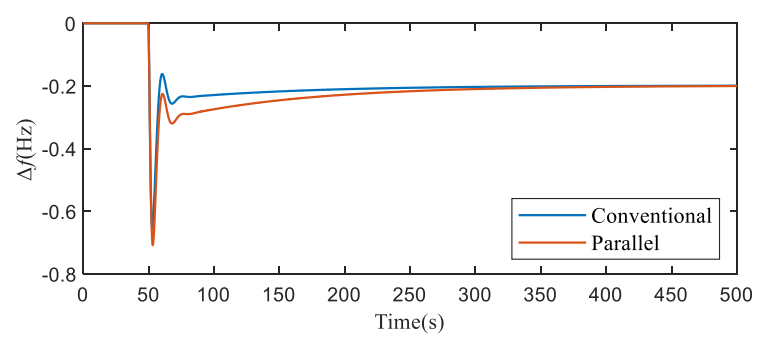

Fig. 9. The frequency response for large disturbance $\left(k_{\mathrm{FCR}-\mathrm{N}}=\right.$ $0.4, k_{\text {FCR-D }}=0$ and $k$ FCR-N/D $=0.6$ )

When the frequency deviation exceeds the dead zone, part of the power support is provided by FCR-N in the parallel method, while all the power support is provided by FCR-D in the conventional method, which makes the regulation speed in the conventional method is faster and the time for the frequency to reach the steady state is shorter.

Assumed $k_{\mathrm{FCR}-\mathrm{N} / \mathrm{D}}=1$, that is to say all the units using switching parameters instead of constant parameters, according to the simulation, the damping of conventional method is larger clearly in Fig. 10.

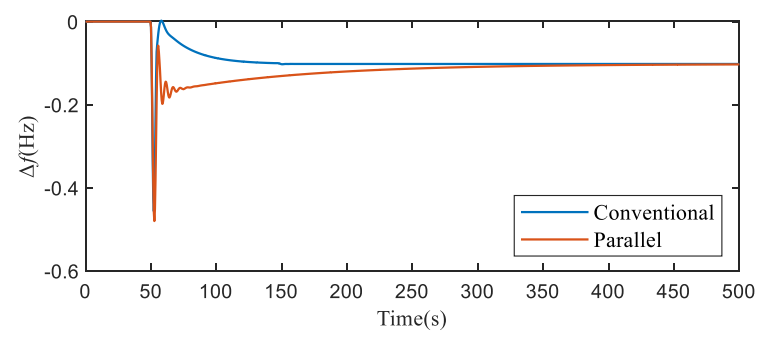

Fig. 10. The frequency response for large disturbance $(k$ FCR-N $=$ $0, k_{\text {FCR-D }}=0$ and $k$ FCR-N/D $\left.=1\right)$

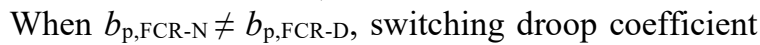
in conventional method can cause serious frequency problems. Compared with the conventional method, the parallel method does not have the problem of switching droop coefficient, while the performance and damping ratio of the parallel method is not as good as that of the conventional method. Therefore, the conventional method of $b_{\mathrm{p}}$ equivalent PID parameter switching is recommended in this paper.

\section{Parameters optimization of the recommended method}

In the one hand, in order to obtain a larger frequency stability margin, the speed regulation performance is sacrificed in FCR-N. In the other hand, the objective of FCR-D is to act rapidly after a large disturbance to pull the frequency back into the dead zone, where parameter settings may make units provide negative damping.

In this section, the parameters are optimized to improve the frequency response characteristic under large disturbance in a single machine system. It is assumed that the conventional method with constant $b_{\mathrm{p}}$ is adopted.

\subsection{Parameters optimization within the dead zone}

The parameters optimization method based on state space and particle swarm optimization is adopted to optimize the parameters within dead zone by considering both the response speed and stability of primary frequency control.

The optimization model can be expressed as: $\min t_{\mathrm{d}}$

subject to

$$
\begin{gathered}
K_{\mathrm{P}-\min } \leq K_{\mathrm{P}} \leq K_{\mathrm{P}-\max } \\
K_{\mathrm{I}-\min } \leq K_{\mathrm{I}} \leq K_{\mathrm{I}-\max } \\
K_{\mathrm{D} \text {-min }} \leq K_{\mathrm{D}} \leq K_{\mathrm{D}-\max } \\
t_{\text {s-min }} \leq t_{\mathrm{s}} \leq t_{\text {s-max }}
\end{gathered}
$$

where $t_{\mathrm{d}}$ is the time required for the frequency response to rise to $80 \%$ of steady value, $t_{\mathrm{s}}$ is the time when the deviation between the frequency response and the steady-state value reaches the allowable range and is set to $\pm 2 \%$.

According to the equations above, the unconstrained comprehensive objective function is obtained through the penalty function. Afterwards, the speed regulation 
parameters are optimized, and the parameters before and after optimization were shown in Table 2.

Table 2. Optimization parameters for FCR-N

\begin{tabular}{|c|c|c|c|c|}
\hline & $\boldsymbol{K}_{\mathbf{P}}$ & $\boldsymbol{K}_{\mathbf{D}}$ & $\boldsymbol{K}_{\mathbf{I}}$ & $\begin{array}{c}\text { response } \\
\text { time } / \mathbf{s}\end{array}$ \\
\hline original & 4.436 & 0 & 0.2 & 360.14 \\
\hline optimized & 6.7 & 2.5 & 0.7 & 118.7 \\
\hline
\end{tabular}

Under the step disturbance, the simulation was shown in Fig. 11. It can be found that the FCR-N optimization parameter in this paper can better respond to the large power shortage, reduce the frequency peak, and quickly recover to the steady state after disturbance.

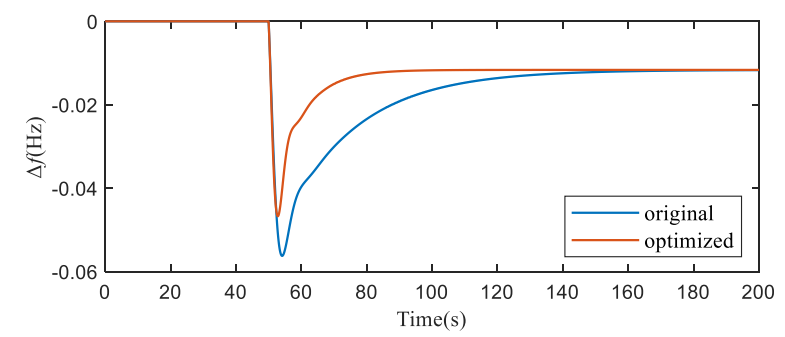

Fig. 11. The frequency response with different parameters for FCR-N

\subsection{Parameters optimization exceed the dead zone}

The goal of FCR-D is to quickly pull the frequency back to the dead zone after a large disturbance, so the damping exceed the dead zone is small. For $T_{\mathrm{w}}$ large hydropower units, the single machine system is likely to be close to zero damping or even negative damping, which may lead to frequency oscillation.

According to the calculation results, when $T_{\mathrm{w}}>1.6 \mathrm{~s}$, the system exceeds the dead zone is negatively damped using the original parameters. If the disturbance is large enough, the system frequency oscillates. It can be inferred that capacity proportion of units with switching parameters increases, the damping of frequency oscillation decreases.

The frequency response is shown in the Fig. 12 when the equivalent $T_{\mathrm{w}}=1.4 \mathrm{~s}$, although the damping is positive, frequency oscillation will occur when FCR-D is regulated too fast and FCR-N cannot balance overshoot in a short time, which means requirements for regulating performance should be relaxed.

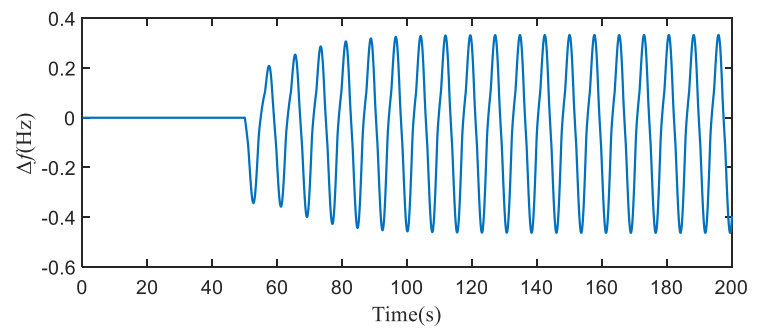

Fig. 12. The frequency response with original parameters for $\mathrm{FCR}-\mathrm{D}\left(k_{\mathrm{FCR}-\mathrm{N}}=0, k_{\mathrm{FCR}-\mathrm{D}}=0\right.$ and $\left.k_{\mathrm{FCR}-\mathrm{N} / \mathrm{D}}=1\right)$

A parameters optimization model for disturbances is proposed to avoid the frequency oscillation: $\min t_{\mathrm{s}}$

subject to

$$
\begin{gathered}
\zeta \geq 0 \\
K_{\text {P-min }} \leq K_{\mathrm{P}} \leq K_{\mathrm{P}-\max } \\
K_{\mathrm{I} \text {-min }} \leq K_{\mathrm{I}} \leq K_{\mathrm{I} \text {-max }} \\
K_{\text {D-min }} \leq K_{\mathrm{D}} \leq K_{\mathrm{D} \text {-max }}
\end{gathered}
$$

where $\zeta$ is the damping ratio.

Table 3. Optimization parameters for FCR-D

\begin{tabular}{|c|c|c|c|c|c|}
\hline & $K_{\boldsymbol{P}}$ & $\boldsymbol{K}_{\boldsymbol{D}}$ & $\boldsymbol{K}_{\boldsymbol{I}}$ & $\begin{array}{c}\text { response } \\
\text { time/s }\end{array}$ & $\begin{array}{c}\text { damping } \\
\text { ratio }\end{array}$ \\
\hline original & 10.436 & 8 & 4 & 23.4 & -0.0052 \\
\hline optimized & 5.2 & 6.6 & 1.3 & 58.8 & 0.2648 \\
\hline
\end{tabular}

The parameters before and after is shown in Table 3 , when the 1.3 times $T_{\mathrm{w}}$ value is taken as the stability margin, which approximately equals to $1.6 \mathrm{~s}$.

The two optimization parameters were compared with the system frequency response under large disturbance with the original default parameters when the equivalent $T_{\mathrm{w}}=1.2 \mathrm{~s}$, as shown in the Fig. 13. It can be found that after parameter optimization, FCR-N and $\mathrm{D}$ coordinate with each other under large disturbance, reduce frequency peak, and quickly return to steady state.

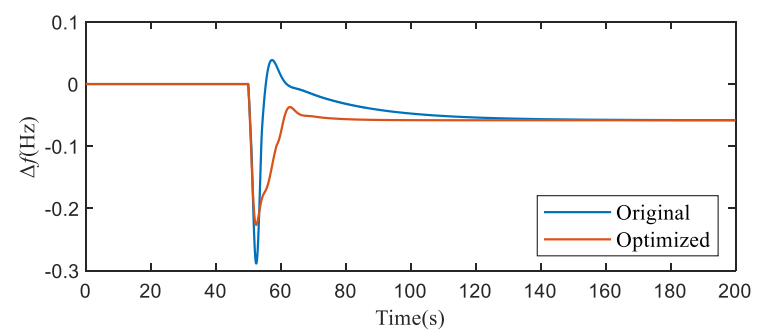

Fig.13. The frequency response with different parameters for $\operatorname{FCR}\left(k_{\mathrm{FCR}-\mathrm{N}}=0, k_{\mathrm{FCR}-\mathrm{D}}=0\right.$ and $k$ FCR-N/D $\left.=1, T_{\mathrm{w}}=1.2 \mathrm{~s}\right)$

\section{Conclusion}

At present, the parameters of the governor of a single hydropower unit may not be able to fulfil the primary frequency control requirements of the power grid and ensure the dynamic stability of the system.

This paper researches the operation characteristics of parameters switching methods. By analyzing, the advantages and disadvantages of conventional method and parallel method are grasped, and the recommended method is proposed. According to the response characteristics and technical requirements of FCR, suggestions to improve FCR were put forward, and the dynamic performance was optimized, which not only improved the response speed of primary frequency control, but also ensured the frequency stability of the system.

Finally, the comparison of primary frequency control before and after optimization verified the effectiveness of the proposed method.

\section{References}

1. J. Zhang, C. Liu, Y. Chen, et al. Southern Power System Technology, Countermeasures and experiments on ultra-low frequency oscillation of 
Yunnan Power Grid in asynchronous interconnect mode. 7, 35-39(2016)

2. C. Xiao, D. Wang, Z. Li, et al. Power System Technology, Research on parameter optimization control of turbine governor for suppressing ultralow frequency oscillation in power systems.1-9(2019)

3. L. Chen, X. Lu, Y. Min, et al. IEEE Trans on Power Systems, Optimization of governor parameters to prevent frequency oscillations in power systems. 33, 4466-4474(2018)

4. H. Shi, G. Chen, L. Ding, et al. Power System Technology, PID parameter optimization of hydro turbine governor considering the primary frequency regulation performance and ultra-low frequency oscillation suppression. 43, 249-254(2019)

5. W. Huang, R. Duan, C. Jiang, et al. Automation of Electric Power Systems, Stability analysis of ultra- low frequency oscillation and governor parameter optimization for multi-machine system. 42, 185191(2018)

6. E. Agneholm, S. Meybodi, Kuivaniemi, et al. ENTSO-E, FCR-D design of requirements-phase 2 (2019)

7. H. Olsson. Uppsala University, Future frequency control services in the Nordic power system (2017)

8. ENTSO-E. ENTSO-E, AS survey results 2016(2017)

9. E. Agneholm, S. Meybodi, Kuivaniemi, et al. ENTSO-E, FCR-Design project summary report(2019)

10. Q. Gao, Q. Liu, Y. Chen, et al. Power System Technology, Influence of different deadbands on the frequency stability (2020) 